\title{
An Approach to Remote Monitoring of Heart Rate Variability (HRV) Using Microwave Radar during a Calculation Task
}

\author{
Satoshi Suzuki ${ }^{1)}$, Takemi Matsui ${ }^{2)}$, Keitaro Sugawara ${ }^{2)}$, Takafumi Asao ${ }^{1)}$ and Kentaro Kotani ${ }^{1)}$ \\ 1) Faculty of System Engineering, Kansai University \\ 2) Faculty of System Design, Tokyo Metropolitan University
}

\begin{abstract}
Recently, nonrestrictive and noninvasive sensing techniques to measure vital signs have been actively researched and developed. This study aimed to develop a prototype system to monitor cardiac activity using microwave radar without making contact with the body and without removing clothing - namely, a completely noncontact, remote monitoring system. In addition, heart rate and changes in heart rate variability (HRV) during simple mental arithmetic tasks were observed with the prototype system. The prototype system has a microwave Doppler radar antenna with $24 \mathrm{GHz}$ frequency and approximately $7 \mathrm{~mW}$ output power. The experiments were conducted with seven subjects ( $23.00 \pm 0.82$ years). We found that the prototype system captured heart rate and HRV precisely. The strong relationship between the heart rates during tasks $(r=0.96)$, LF (cross-correlation $=0.76)$, and $\mathrm{LF} /$ HF (cross-correlation $=0.73$ ) of HRV calculated from the prototype system and from electrocardiograph (ECG) measurements were confirmed. The proposed completely noncontact, remote method appears promising for future monitoring of cardiac activity as an indicator of changes in mental workload in workplaces. J Physiol Anthropol 30(6): 241-249, 2011 http://www.jstage.jst.go.jp/browse/jpa2
\end{abstract}

[DOI: 10.2114/jpa2.30.241]

Keywords: noncontact, microwave radar, heart rate variability (HRV), remote monitoring, calculation task

\section{Introduction}

In order to design a job and a workplace appropriately, it is important to reduce extremely heavy workloads as well as to prevent the occurrence of health problems and minimize human error. In general, for the evaluation of mental workload, in all its multidimensionality, it is necessary to study employees from subjective, objective, and physiological perspectives (Johannsen et al., 1979). There has been much discussion about mental and physical workloads at TC159/SC1 of ISO, and international standards have been developed (ISO
10075-2, 1996; ISO 10075-3, 2004). The TC159/SC1 offers guidelines on dealing with problems associated with mental workloads. Many subjective assessment methods like the NASA-TLX (Hart and Stave, 1988) have been developed and are frequently used to estimate mental workload while working (Averty et al., 2004; Bunce and Sisa, 2002). However, in order to prevent traffic and industrial accidents, developing an assessment method that is more objective and quicker to complete is desirable. It is thought that one of the most useful methods of evaluating mental workload is the monitoring of changes in the autonomic nervous system by measuring heart rate variability (HRV) (Derrick, 1988). Many approaches to measuring HRV are used in medical treatment (Singh et al., 1996; Carney et al., 2001), psychology (Vincent et al., 1996), occupational health (Miyake, 2001; Princi et al., 2005), and ergonomics research (Sirevaag et al., 1993; Gould et al., 2009). Such monitoring has led to significant progress in medical diagnosis and research. Monitoring HRV is a reliable and wellestablished method of evaluating mental workload; however, examples of daily use in real workplaces are extremely few. It is thought that the main problem with such monitoring has to do with its practicality, and this is partly due to restriction of motion and the complications of placing electrodes directly on the skin. Long-term electrocardiographic (ECG) monitoring using electrodes places a heavy burden on monitored individuals.

Recently, nonrestrictive and noninvasive sensing techniques to measure vital signs have been actively researched and developed. Examples include using a strain gauge (Ciaccio et al., 2007), pressure sensors (Jacobs et al., 2004), and a piezoelectric polymer called polyvinylidene fluoride film (PVDF) in sensors (Wang et al., 2006) used to measure heartbeat and respiration from the subject's dorsal body surface. This kind of sensing technique is useful for patients who have suffered heavy burn injuries and serious lacerations, because it avoids having to paste electrodes directly onto the body. Furthermore, patients who should be isolated because of the risk of secondary infection resulting from exposure to toxic chemicals or infectious organisms are treated in an isolator and 
in such cases a stable remote sensing method is needed to measure vital signs from outside of the isolator.

We have previously reported on a complete noncontact system for monitoring respiratory rate and heart rate using a microwave radar antenna at $1215 \mathrm{MHz}$ to measure the vital signs of casualties inside an isolation unit (Matsui et al., 2004; Matsui et al., 2006). In addition, we developed a noncontact method using a ceiling-mounted microwave radar to monitor the respiratory rates of subjects in bed through their bedding (Uenoyama et al., 2006). These methods are aimed at detecting motion at extremely minute scales on the body surface caused by cardiac and respiratory motion. The method was originally developed to search for survivors under earthquake rubble (Chen et al., 1986; Chen et al., 2000). Microwave radar has the following characteristics: (1) microwaves can be transmitted through most objects except metals and water; and (2) it is possible to detect movement of the object from some distance and without actually needing to touch it. If we attempt to use this system for humans, it is possible to observe the motion of the body surface from some distance without removing their clothing.

We have already tried to monitor changes in HRV that are induced by stressful audio stimuli using a noncontact measurement system with a $24 \mathrm{GHz}$ compact microwave radar, which can easily be attached to the rear surface of the back of a chair (Suzuki et al., 2008a). The aim of this study was to develop a prototype of the same type of system for cardiac monitoring, using microwave radar without making contact with the body and without removing clothing-namely, a complete noncontact, remote monitoring system. In addition, heart rate and changes in heart rate variability during tasks were measured by utilizing the system.

\section{System Design of a Prototype System Using Microwave Radar for Noncontact Cardiac Monitoring}

\section{Microwave Doppler radar and its specifications}

The prototype system for noncontact cardiac monitoring we designed consisted of a microwave Doppler radar antenna (TAU GIKEN Co., Yokohama, Japan), a device for controlling the power supply to this antenna, and a PC for analyzing the output data from the antenna. The frequency of this microwave radar antenna for cardiac monitoring was $24 \mathrm{GHz}$, with a normal average output power of approximately $7 \mathrm{~mW}$ (the maximum output power is under $10 \mathrm{~mW}$ ). The diffusion angle $\left(\theta_{d}\right)$ of the microwave radar antenna is about $40^{\circ}$, the antenna gain is $10 \mathrm{dBi}$, and the electrical field intensity is $0.7 \mathrm{~mW} / \mathrm{cm}^{2}$. This antenna has approximately the same specifications as that used in our previous research (Suzuki et al., 2008a; Suzuki et al., 2009).

Damage caused by electromagnetic waves has been discussed in the literature, especially in the case of human applications. At frequencies over $3 \mathrm{GHz}$, the electrical field intensity limit is $1 \mathrm{~mW} / \mathrm{cm}^{2}$ according to the guidelines for radio waves established by the Telecommunication Bureau of the Ministry of Internal Affairs and Communication in Japan. The electrical field intensity of this microwave radar is 0.7 $\mathrm{mW} / \mathrm{cm}^{2}$; it is therefore in conformity with the guideline. Furthermore, the $24 \mathrm{GHz}$ frequency of our device is within the frequency band for normal use of radio waves as approved by Japanese law.

\section{System design of prototype system for noncontact cardiac monitoring}

Before input into a PC for analysis, data were acquired at a sampling frequency of $100 \mathrm{~Hz}$ using an A/D converter (USB6008, National Instruments, Texas, USA). After digitization, the data were analyzed by a system we developed using analyzing software (LabVIEW, National Instruments, Texas, USA). In this analyzing system, in order to reduce noise and select data related to the motion associated with heart rate, a band-pass filter was used for transferring data from the microwave Doppler radar antenna. This filter was set between $0.5 \mathrm{~Hz}$ and $2.5 \mathrm{~Hz}$; this setting covers a range of 30 to 150 heartbeats per minute.

\section{Experiment 1: Test of Noncontact Cardiac Monitoring Using the Prototype System}

\section{Methods and procedure}

The prototype system is designed to monitor extremely small movements appearing on the body surface as a result of cardiac motion. In order to verify the effectiveness of the prototype system, we conducted a simple test to measure the motion on the surface of the dorsal surface of the subject's body using a high-resolution laser distance meter (see Fig. 1), and we also used an ECG as a reference, which obtained measurements with normal electrodes attached directly to the body $\left(\mathrm{V}_{5}\right.$ position of precordial $\left.\mathrm{ECG}\right)$.

The prototype system was tested with seven healthy male subjects (mean age 23.00 \pm 0.82 years; range 22-24 years). Table 1 shows data for each subject. At first, subjects stripped to the waist, sat directly on the floor and were encouraged to rest for a while. After resting, the subjects remained seated and data acquisition was started, continuing for 2 minutes. The prototype system was set up about $30 \mathrm{~mm}$ from the body and about $60 \mathrm{~mm}$ to the left of the spine at around the level of the fourth intercostal space. In addition, to measure the motion on the body surface directly and to use the results as a reference, the high-resolution laser distance meter (LK-G35, Keyence Co., Tokyo, Japan) was placed about $60 \mathrm{~mm}$ to the right of the spine at around the level of the fourth intercostal space (see Fig. 1). High-resolution laser distance meters were originally intended for industrial use. The wavelength is $650 \mathrm{~nm}$ and the output power is $4.8 \mathrm{~mW}$. This distance meter is able to measure $2.5 \mu \mathrm{m}$. The laser itself is a Class IIIa/3R device, according to a laser safety standard (JIS C 6802, 2011). However, when used for only a few minutes at a time, it does not affect the body surface because its output power is relatively low. Although noninvasive, we used vinyl stretch tape to protect the 


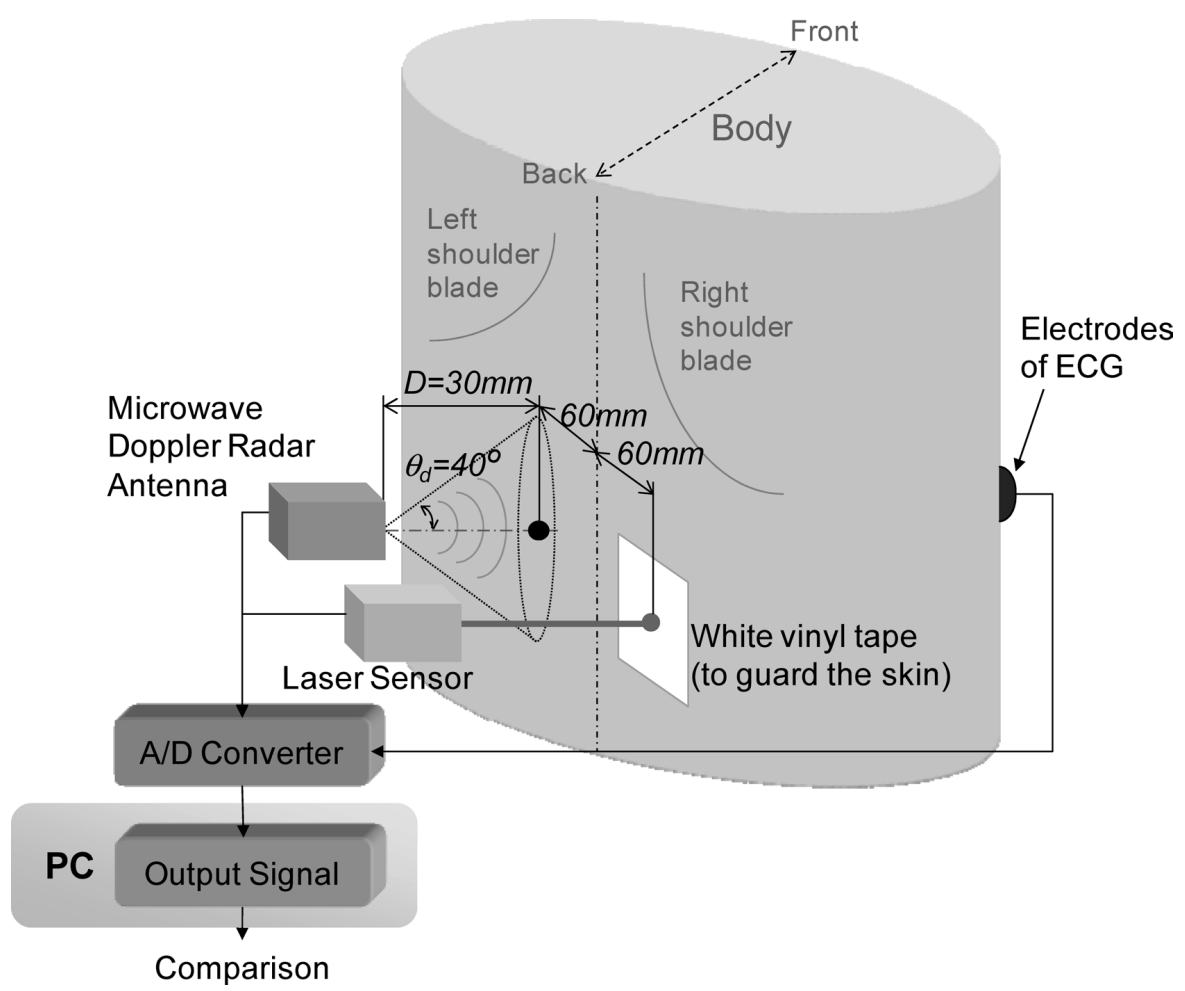

Fig. 1 Schematic diagram of Experiment 1, the experimental setup to test the prototype system for noncontact cardiac monitoring. In order to verify the prototype system, a high-resolution laser distance meter was used to measure the motion on the surface of the dorsal side of the body, and an ECG was also used as a reference, providing direct measurements with a contact monitoring system with normal electrodes attached directly to the body.

Table 1 Individual data on the physical characteristics of seven volunteer subjects ${ }^{\mathrm{a}}$

\begin{tabular}{|c|c|c|c|c|c|c|c|}
\hline \multirow{2}{*}{ Subject } & \multirow{2}{*}{ Age } & \multirow{2}{*}{$\begin{array}{c}\text { Height } \\
(\mathrm{cm})\end{array}$} & \multirow{2}{*}{$\begin{array}{l}\text { Weight } \\
\text { (kg) }\end{array}$} & \multicolumn{2}{|c|}{ Blood pressure } & \multirow{2}{*}{$\begin{array}{c}\text { Backfat } \\
\text { thickness } \\
(\mathrm{mm})\end{array}$} & \multirow{2}{*}{ BMI } \\
\hline & & & & Systolic & Diastolic & & \\
\hline$S_{1}$ & 22 & 181 & 72 & 131 & 80 & 10 & 21.98 \\
\hline$S_{2}$ & 24 & 168 & 59 & 111 & 63 & 6 & 20.90 \\
\hline$S_{3}$ & 23 & 171 & 58 & 101 & 59 & 7 & 19.84 \\
\hline$S_{4}$ & 23 & 170 & 60 & 118 & 50 & 4 & 20.76 \\
\hline$S_{5}$ & 22 & 181 & 67 & 103 & 51 & 5 & 20.45 \\
\hline$S_{6}$ & 23 & 171 & 63 & 125 & 71 & 7 & 21.55 \\
\hline$S_{7}$ & 24 & 170 & 57 & 107 & 58 & 5 & 19.72 \\
\hline Average & 23.00 & 173.14 & 62.29 & 113.71 & 61.71 & 6.29 & 20.74 \\
\hline$S D$ & 0.82 & 5.46 & 5.47 & 11.35 & 10.77 & 1.98 & 0.83 \\
\hline
\end{tabular}

${ }^{a}$ Mean age was $23.00 \pm 0.82$ years (range $22-24$ years). $S D$ : standard deviation.

skin as a precaution. The laser carries a low risk of eye damage within the short time before a blink reflex. Therefore, to ensure safety, all subjects wore safety spectacles (S-80, Riken Optech Co., Tokyo, Japan), which reduce transmittance to $0.1 \%$, and were told to close their eyes unless directed otherwise. The heartbeat was also monitored by an ECG (Web-5500, Nihonkoden Co., Tokyo, Japan) as a reference using a contact monitoring method. The output signals from the three systems were sampled by the $\mathrm{A} / \mathrm{D}$ converter with a sampling frequency of $100 \mathrm{~Hz}$, and all data were saved on a PC.

\section{Results \\ Oscillation}

Figure 2 shows sample data for subject $S_{1}$ monitored by the three measurement systems for $5 \mathrm{~s}$, extracted from the total 2 min of data. Figure 2(A) shows the output signal of the ECG, Fig. 2(B) shows the output signal acquired by the highresolution laser distance meter, and Fig. 2(C) shows the output signal acquired by the prototype system.

As can be seen in Fig. 2(B), cyclic oscillation synchronizing with the occurrence of the R wave of the ECG (Fig. 2(A)) was 
(A)

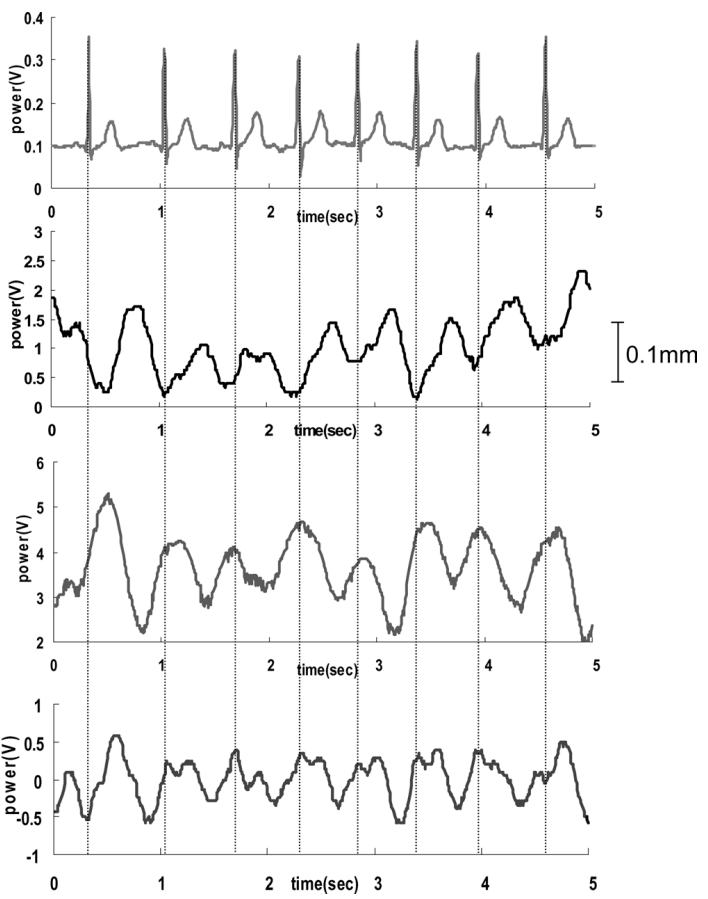

Fig. 2 Sample data for subject $S_{1}$ monitored by three measurement systems in a 5 -second period. (A) The output signal of the ECG; (B) the output signal acquired by the high-resolution laser distance meter; (C) the output signal acquired by the prototype system for noncontact monitoring; (D) the results of the differentiated data of output signal (B) acquired by the laser distance meter.

confirmed. This result means that the body surface periodically vibrates in synchrony with the heartbeat and the amplitude is approximately $0.2 \mathrm{~mm}$ on average. Although there were individual differences, an amplitude of about $0.2 \mathrm{~mm}$ was confirmed in each subject.

Figure 2(C) shows the data for the prototype system. While a phase shift between the cyclic oscillations acquired by the laser distance meter and those acquired by the prototype system was confirmed, the repeat cycles of the two systems were nearly identical. This shift seems to be due to differences in dimension. The laser distance meter is a displacement sensor, and the prototype system measures changes in velocity because a Doppler radar antenna is used. If the data acquired with the laser distance meter are differentiated, the dimension is the same as for the data from the Doppler radar antenna. Figure 2(D) shows the results of differentiation of the data for the laser distance meter (Fig. 2(B)). By comparison, we could not confirm the phase shift between the data acquired by the prototype system (Fig. 2(C)) and the differentiated data from the laser distance meter (Fig. 2(D)), and we can see a similar change.

\section{Cross-correlation}

In order to confirm the similarity of the two methods, the cross-correlation values for the output data from the prototype system and the differentiated data for the laser distance meter

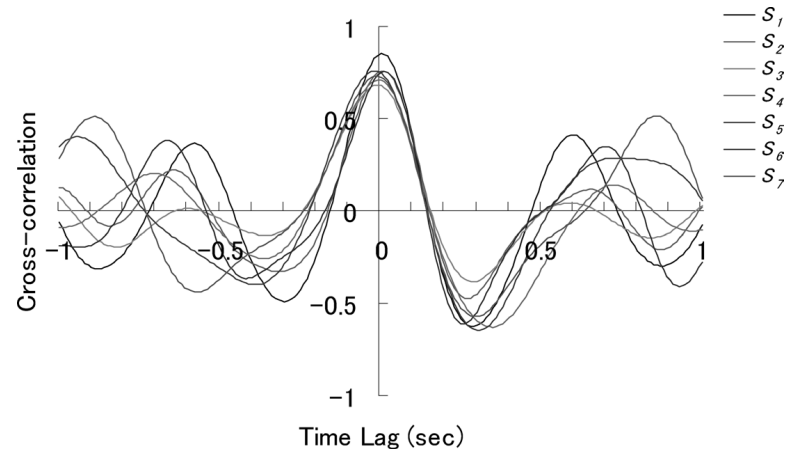

Fig. 3 Cross-correlation values for the output data from the prototype system for noncontact cardiac monitoring and the differentiated data from the laser distance meter for each subject. The cross-correlation values were the highest at about time 0 for all subjects. In addition, the maximum values for all subjects at time 0 were relatively high0.8 on average. These results mean that the prototype system precisely captured the movement on the body surface.

were calculated for each subject (see Fig. 3). From this figure, we can confirm that the cross-correlation values were the highest at about time 0 for all subjects. This result means that a retardation in phase between the two output signals did not exist. In addition, the maximum values for all subjects at time 0 were relatively high -0.8 on average. Therefore, the prototype system precisely captured the movements on the body surface.

\section{Experiment 2: Monitoring Changes in HRV with the Prototype System}

\section{Task procedure and settings}

Experiments to measure heart rate and changes in HRV using the prototype system used in Experiment 1 were conducted. At the same time, the ECG was measured by the contact monitoring system using normal electrodes. We compared the results for the ECG with the results acquired by the prototype system (see Fig. 4).

The same subjects as participated in Experiment 1 were employed for Experiment 2. Each subject wore a $2 \mathrm{~mm}$-thick cotton T-shirt and sat on a chair with mesh back composed of 2 mm-thick polyester plastic (Baron-Chair, Okamura Co., Tokyo, Japan). The distance of the antenna of the prototype system to the chair back was $30 \mathrm{~mm}$, and it was placed about $60 \mathrm{~mm}$ to the left of the spine at around the level of the fourth intercostal space- the same position as for Experiment 1.

Following a period of 2 minutes' silence for resting, the subjects were asked to perform a simple arithmetic task with pairs of two-digit numbers for a period of $2 \mathrm{~min}$. Two-digit numbers in randomly produced pairs were displayed on a personal computer screen. The subject calculated in his head and inputted the answer from the ten-key keyboard within $3 \mathrm{~s}$.

The distance from the display to the subject's face was fixed at $700 \mathrm{~mm}$, the height of the desktop was $700 \mathrm{~mm}$ from the floor, and the height of the seat from the floor was $400 \mathrm{~mm}$. 


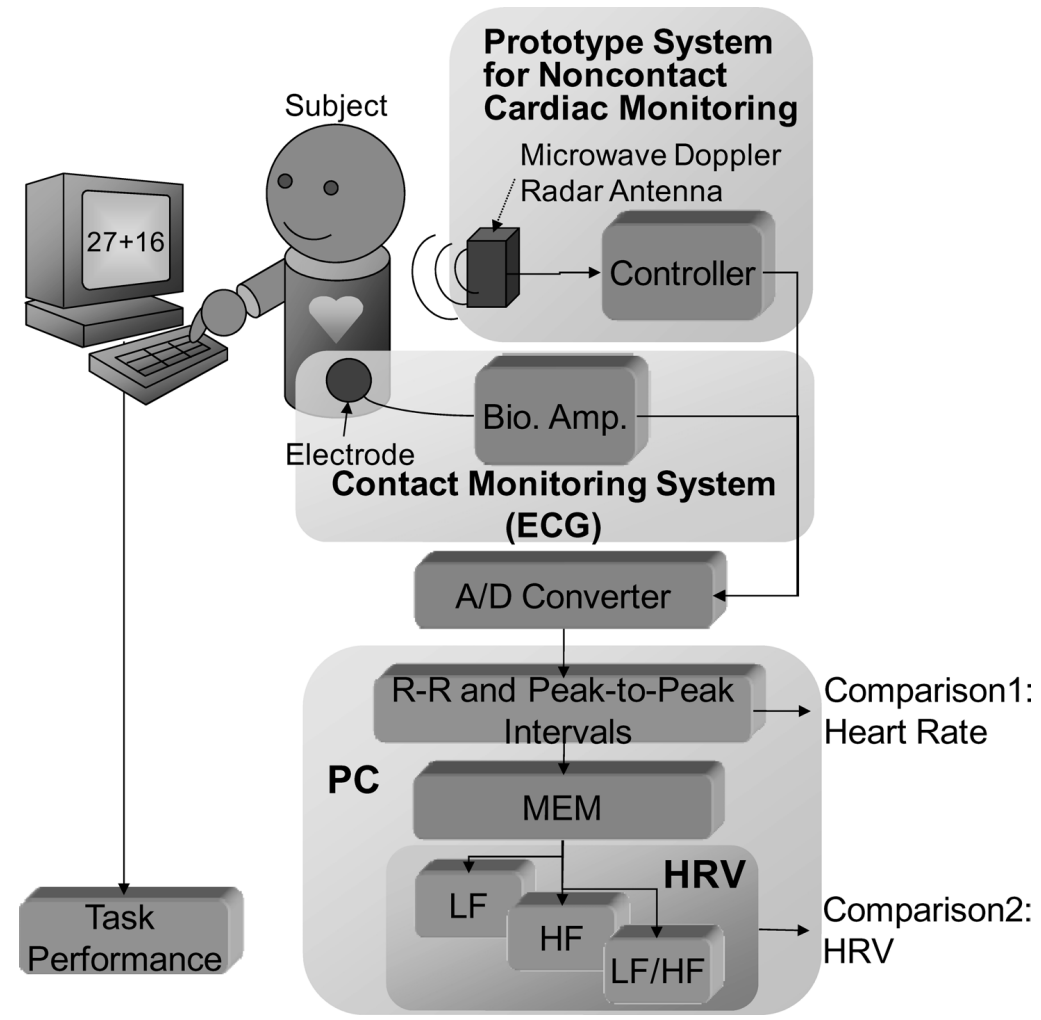

Fig. 4 Schematic diagram of Experiment 2, which measured heart rate and changes in HRV using the prototype system for noncontact cardiac monitoring. The experiment was conducted during mental arithmetic tasks with pairs of two-digit numbers and input into a computer. In order to compare these results with a reference, an ECG was simultaneously used.

The subject rested his right elbow on the armrest of the chair and was directed to keep it there when he inputted the answers using the keyboard. All subjects were right-handed. We did not give the subjects any instructions on breathing, such as holding the breath, and informed consent was obtained from each subject. All study protocols were reviewed and approved by the institutional committee on human studies (Faculty of System Design, Tokyo Metropolitan University, Tokyo, Japan).

\section{Analysis}

The output signals from the prototype system and a reference precordial ECG signal from the $\mathrm{V}_{5}$ position were sampled by the A/D converter with a sampling frequency of $100 \mathrm{~Hz}$. Band-pass filters were used for the prototype system outputs to reduce noise and interference. The band-pass filters were set at between $0.5 \mathrm{~Hz}$ and $2.5 \mathrm{~Hz}$; this model band-pass filter covers a range of 30 to 150 heartbeats per minute. After the filtering, the power spectra of heartbeat intervals - as low frequency (LF) (0.04-0.15 Hz), high frequency (HF) (0.15-0.4 $\mathrm{Hz}$ ), and LF/HF (Singh et al., 1996; Carney et al., 2001)were calculated to monitor HRV by using the maximum entropy method (MemCalc software, GMS Co., Tokyo, Japan); this method is normally used for medical research (Clayton et al., 1995; Suzuki et al., 2008b).

The intervals of the peaks in amplitude in outputs from the prototype system were assumed to correlate with the $\mathrm{R}-\mathrm{R}$ interval for the ECG, and HRV was calculated by using peakto-peak intervals in the output signal of the prototype system. The power spectra of HRV (i.e., LF, HF, and LF/HF) for R-R intervals derived by the ECG were also calculated by using the MemCalc software. Cross-correlations were examined for the $\mathrm{LF}, \mathrm{HF}$, and LF/HF derived from our noncontact system and the LF, HF, and LF/HF derived from the contact ECG system. Quantitative data are expressed as mean $\pm S D$. Sample size was determined to achieve sufficient assurance for the paired t-test for relatively uniform subjects.

\section{Results}

\section{Heart rate}

Figure 5(A) shows comparisons between the heart rates determined by the prototype system and those obtained by the reference ECG during the silent period of 2 minutes' rest before the tasks were performed. The horizontal axis indicates the $\mathrm{R}-\mathrm{R}$ interval in the data derived from the normal ECG. The vertical axis indicates the peak-to-peak interval estimated by using the data from the prototype system using microwave Doppler radar. A strong positive correlation $(r=0.95)$ in the two indices was confirmed for all subjects.

Figure 5(B) shows the correlation between the peak-to-peak interval determined by the prototype system and the $\mathrm{R}-\mathrm{R}$ interval determined by the normal ECG during the performance of the arithmetic and computer input task 


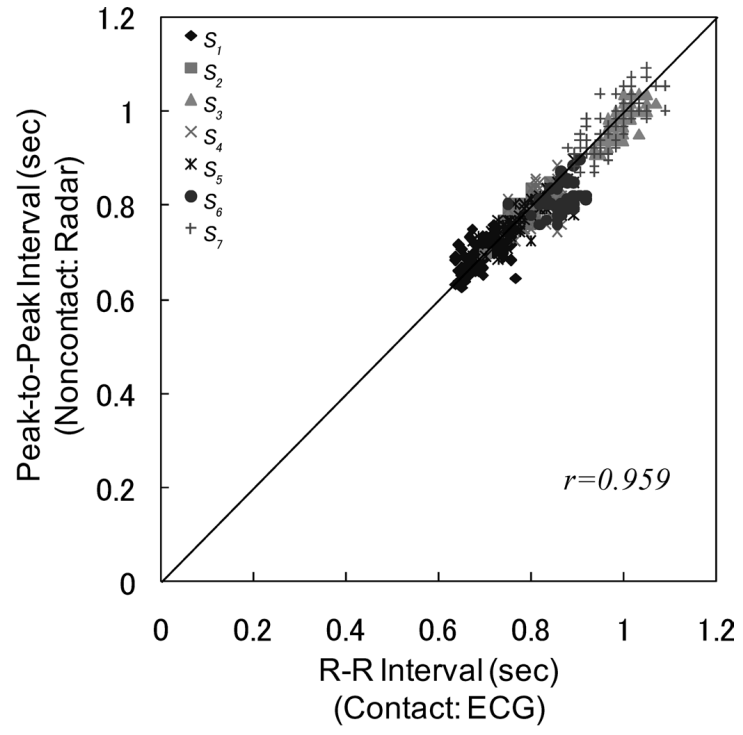

(A)

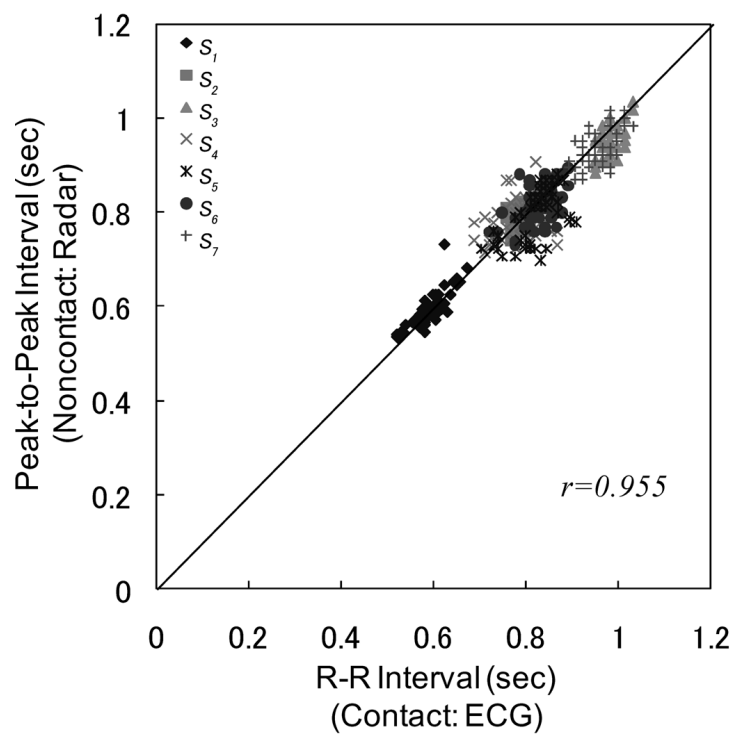

(B)

Fig. 5 Correlation diagrams for both the noncontact and contact monitoring methods under two conditions of resting and task performance. (A) Results of comparison between the peak-to-peak interval determined by the prototype system for noncontact cardiac monitoring and the R-R interval determined by the ECG as a reference for the period of 2 minutes' silence for rest before the task. An extremely positive correlation in the two indices was confirmed for all subjects $(r=0.954)$. (B) Results for the peak-to-peak interval derived by the noncontact and the R-R interval derived by the contact monitoring systems during task performance. The prototype system was also able to capture heart rate with a high correlation $(r=0.963)$ during task performance.

$(r=0.96)$. The high correlation between the heart rates derived by the two systems was confirmed for all subjects irrespective or whether the subjects were performing the task or resting. Therefore, the prototype system measures heart rate stably and independent of the subjects' task.

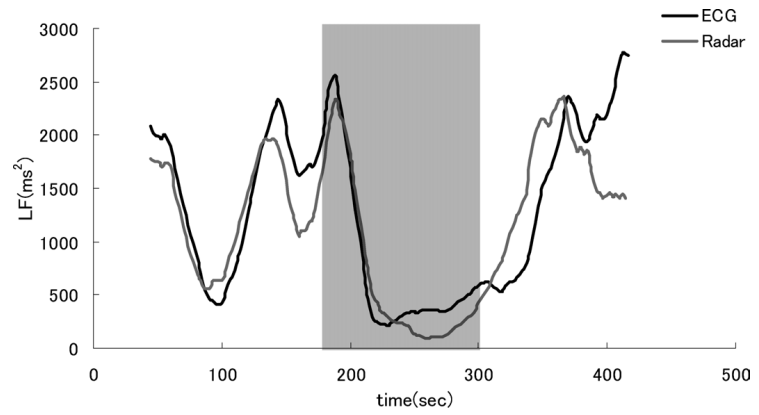

(A)

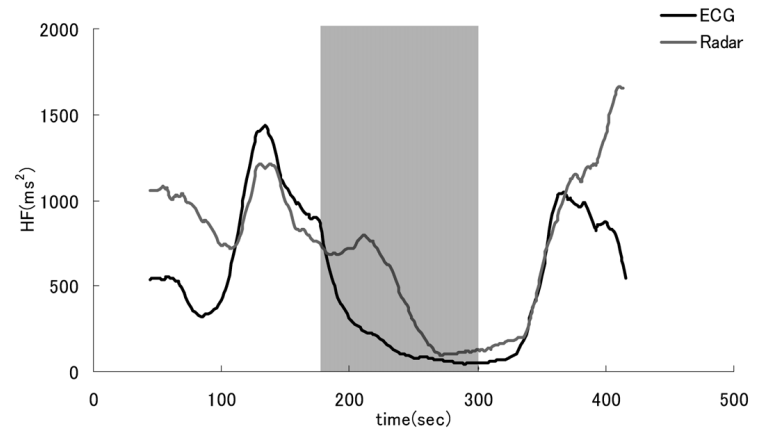

(B)

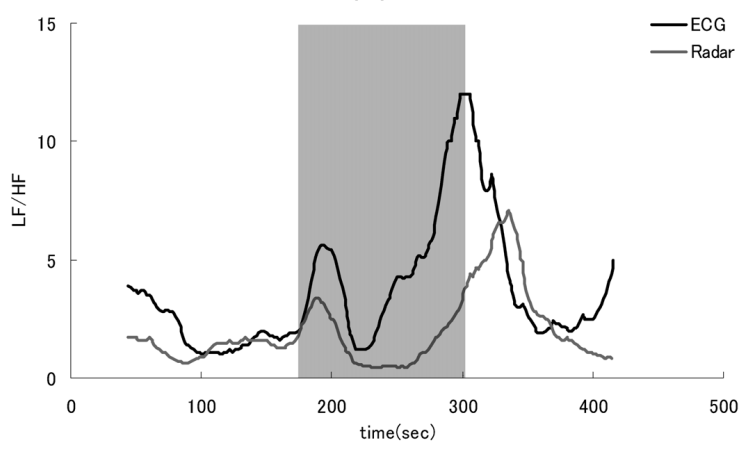

(C)

Fig. 6 Sample data for subject $S 1$ showing changes in HRV for the noncontact and contact monitoring systems. (A) The HRV parameter LF (reflecting mainly sympathetic activation) showed similar changes in the 2-minute silent rest period before the tasks and during task performance. (B) The HF for the same subject (reflecting parasympathetic activity) did not show any distinctive change during the mental arithmetic and computer input task. (C) The LF/HF of the same subject (reflecting sympathovagal balance) exhibited a peak during the task.

Changes in HRV and cross-correlation between the contact and noncontact monitoring systems

For both the prototype system using microwave radar for noncontact cardiac monitoring and the normal ECG system for contact monitoring as a reference, the HRV parameter LF for subject $S_{1}$ reflected mainly sympathetic activation (Fig. 6(A)); they both showed a similar change at the 2-minute rest period and during the performance of the task. The HF for the same subject, reflecting parasympathetic activity, did not show any distinctive change during the mental arithmetic and computer input task (Fig. 6(B)). The LF/HF for the same subject, 
Table 2 Results of cross-correlation of HRV for seven subjects measured by both the noncontact and contact monitoring systems during rest and task performance ${ }^{b}$

\begin{tabular}{cccc}
\hline Subject & LF & HF & LF/HF \\
\hline$S_{1}$ & 0.84 & 0.68 & 0.74 \\
$S_{2}$ & 0.75 & 0.48 & 0.73 \\
$S_{3}$ & 0.64 & 0.81 & 0.76 \\
$S_{4}$ & 0.66 & 0.57 & 0.46 \\
$S_{5}$ & 0.95 & 0.55 & 0.96 \\
$S_{6}$ & 0.69 & 0.68 & 0.70 \\
$S_{7}$ & 0.76 & 0.30 & 0.79 \\
\hline Average & 0.76 & 0.58 & 0.73 \\
SD & 0.11 & 0.16 & 0.15 \\
\hline
\end{tabular}

${ }^{\mathrm{b}}$ Maximum cross-correlation values for LF for the noncontact and contact methods averaged $0.76 \pm 0.11$ for the seven subjects. Maximum crosscorrelation values for $\mathrm{HF}$ averaged $0.58 \pm 0.16$ for the seven subjects, and maximum cross-correlation values for $\mathrm{LF} / \mathrm{HF}$ averaged $0.73 \pm 0.15$ for the seven subjects. $S D$ : standard deviation.

reflecting sympathovagal balance, exhibited a peak during the task (Fig. 6(C)).

Table 2 shows the results of cross-correlations of HRV for the seven subjects monitored by the noncontact and contact methods during rest and task performance. Maximum crosscorrelation values in LF between the noncontact and contact methods averaged $0.76 \pm 0.11$ for the seven subjects. Maximum cross-correlation values in $\mathrm{HF}$ averaged $0.58 \pm 0.16$ for the seven subjects, and maximum cross-correlation values of $\mathrm{LF} / \mathrm{HF}$ averaged $0.73 \pm 0.15$ for the seven subjects.

\section{Discussion}

System design of the prototype system for noncontact cardiac monitoring

We developed a prototype system using $24 \mathrm{GHz}$ microwave radar for noncontact cardiac monitoring. Compared with other noninvasive measurement methods (i.e., strain gauge (Ciaccio et al., 2007), pressure sensors (Jacobs et al., 2004), and PVDF sensors (Wang et al., 2006), our method is completely noncontact and furthermore does not require the removal of clothing. We designed the antenna with relatively small dimensions to obtain high gain with high spatial resolution; the small size also reduces the possibility of signal absorption through the human body, at $24 \mathrm{GHz}$ more than with lower frequencies. In addition, a high gain allows a smaller area to be analyzed. As a result, a small antenna at $24 \mathrm{GHz}$ is easier to integrate into a monitoring system and is suitable for civil applications. The device can be produced at a low cost, which makes it competitive, when produced on a large scale. On the other hand, the signal processing required is delicate, because there is a space between the body and the microwave radar antenna and it is susceptible to other body motions. These disadvantages are compensated for by the relatively high antenna gain at $24 \mathrm{GHz}$, which makes better spatial resolution possible.

\section{Implications of Experiment 1 Results}

In Experiment 1, we confirmed that the body surface periodically vibrates when the heart beats, and the level of vibration is about $0.2 \mathrm{~mm}$. In addition, our prototype system was able to capture small vibrations appearing on the body surface as cyclic oscillations that correspond to cardiac motion. While a phase shift was confirmed, the repeat cycles were identical. The output signals acquired from our prototype system have a high cross-correlation with the signals acquired from the contact ECG method. Therefore, it is thought that our prototype system succeeded in capturing cardiac motion without making direct contact with the subject's body.

The peaks in the output signal acquired from the prototype noncontact cardiac monitoring system were delayed compared with the R-waves in the ECG signal; the shape, however, appeared to be similar to the pulse wave or change in blood pressure. Generally, ventricular systole occurs from the top of the R-wave to near the end of the T-wave. The aortic valve is opened near the end of the S-wave. Therefore, blood pressure increases between the S- and T-waves (O'Rourke et al., 1992), and it is thought that the peak of the motion, at extremely minute scales, appears on the body surface at the same time. This conclusion indicates that the noncontact system was able to obtain similar information on the $\mathrm{R}-\mathrm{R}$ interval by detecting these peaks.

\section{Implications of Experiment 2 Results}

In order to evaluate the relationship between the intervals detected between peaks in the output signal acquired from the prototype system for noncontact cardiac monitoring and the $\mathrm{R}-\mathrm{R}$ intervals measured by the ECG, the correlation of heart rates obtained with the two methods was calculated. The results confirmed that peak-to-peak intervals captured by our prototype system are similar to the $\mathrm{R}-\mathrm{R}$ intervals captured by the ECG signal, because the estimated heart rates determined by using the data captured by our prototype system are roughly in accordance with actual measurements. Results confirmed that our microwave system can capture information similar to that obtained with the ECG system.

In addition, the changes in HRV measured by both methods were also similar, although there were some differences in the absolute values. These results mean that our prototype system can capture signals with sufficient accuracy to calculate heart rate and HRV. This success is attributed to improved resolution with the higher-frequency microwave radar. Other research has aimed at capturing heart rate (Lohman et al., 2001; Thijs et al., 2005); the frequencies used in those studies were from 1.6 to $2.4 \mathrm{GHz}$. In order to observe changes in HRV, accurate R-R intervals have to be captured. Our prototype system has a higher-frequency microwave radar antenna with a $24 \mathrm{GHz}$ frequency and has the advantages of higher resolution and noninvasiveness.

However, the signals for some subjects were sometimes 
distorted during task performance. It is thought that this noise was caused by motions of the upper limbs induced by task performance. We predicted before the experiments that signals would be affected by upper limb motion, because there is some space between the body and the microwave radar antenna, and the device can be affected by other body motions. However, this noise was observed in only some of the data during task performance, and high values of cross-correlation between HRV measured by the noncontact and contact methods during task performance in all subjects were confirmed. Future work should aim to resolve this problem by using filters with appropriate settings or by interpolation.

At the start of this research, we thought that the sympathetic nervous activation related to task performance could be shown-namely, by LF and/or LF/HF increases or HF decreases. The results confirmed that $\mathrm{LF} / \mathrm{HF}$ increased in many subjects; however, a trend of decreasing LF was confirmed for many subjects (see Fig. 6(A)). It is thought the task difficulty and the 3-s interval set for each trial were not appropriate. It will be necessary to set a time limit or time pressure for each trial in future experiments.

\section{Conclusion}

We describe here a novel prototype system using microwave radar for noncontact cardiac monitoring that requires neither direct contact with the body nor the removal of clothing. We monitored changes in HRV during task performance with the system in efforts to monitor human autonomic activation induced by task performance.

The antenna of our prototype system is relatively small and can easily be attached to office furniture in the workplace (i.e., to a chair back or chair arm). This means that the device is suitable for civil applications at a low cost. We will examine actual use in office settings in the future.

The device is sensitive to other body motions since there is a space between the body and the microwave radar antenna. The signal processing becomes more delicate in the case of work associated with relatively large body motions. Therefore, there are many issues that need to be tackled before using this remote sensing technique in the real workplace; this technique is still in an early phase of research. However, if it becomes possible to use it in the workplace, heart rate can be monitored without large-scale equipment and without placing a heavy burden on the monitored individual. Therefore, large populations can be rapidly investigated. This makes the technique favorable for epidemiological surveillance and massmedicine. In fact, we have already attempted to apply this technique for screening of individuals with suspected infection at a quarantine depot (Matsui et al., 2009, 2010). We have already succeeded in diagnosing influenza patients in as little as $10 \mathrm{~s}$ per person. Our sensing technique may contribute to further developments in population biology and also to applications and findings in physiological anthropology.

In addition, this technique of acquiring data related to cardiac activity without the need for direct contact with the body should contribute greatly to ensuring safety in the workplace since it can contribute to research in the areas of ergonomics and occupational health. Cardiac function is also an important parameter for investigation and evaluation in the field of physiological anthropology. For examples, HRV is used for investigating the effects of reward and punishment with respect to particular personality types (Sakuragi and Sugiyama, 2009) and for investigating inter- and intraindividual variations in Japanese males (Kobayashi, 2007). Additionally, characteristics of HRV changes by age and cardiac autonomic modulation would presumably be maturated before the age of approximately 7-8 years (Fukuba et al., 2009). Therefore, our method appears to be promising, not only for use in ergonomics and physiological anthropological research, but also in a number of other fields.

Acknowledgment The present study was supported by a Grant-in-Special Aid for Promotion of Research and Education (Reconstruction from Earthquake Disasters) founded by Kansai University.

\section{References}

Averty P, Collet C, Dittmar A, Athènes S, Vernet-Maury E (2004) Mental workload in air traffic control: An index constructed from field tests. Aviat Space Environ Med 75: 333-341

Bunce D, Sisa L (2002) Age differences in perceived workload across a short vigil. Ergonomics 45: 949-960

Carney RM, Blumenthal JA, Stein PK, Watkins L, Catellier D, Berkman LF, Czajkowski SM, O’Connor C, Stone PH, Freedland KE (2001) Depression, heart rate variability, and acute myocardial infarction. Circulation 104: 2024-2028

Clayton RH, Bowman AJ, Ford GA, Murray A (1995) Measurement of baroreflex gain from heart rate and blood pressure spectra: A comparison of spectral estimation techniques. Physiol Meas 16: 131-139

Chen KM, Misra D, Wang H, Chuang HR, Postow E (1986) An $\mathrm{X}$-band microwave life-detection system. IEEE Trans Biomed Eng 33: 697-702

Chen KM, Huang Y, Zhang J (2000) Microwave life-detection systems for searching human subjects under earthquake rubble or behind barrier. IEEE Trans Biomed Eng 27: 105-113

Ciaccio EJ, Hiatt M, Hegyi T, Drzewiecki GM (2007) Measurement and monitoring of electrocardiogram belt tension in premature infants for assessment of respiratory function. Biomed Eng Online 6: 1-11

Derrick WL (1988) Dimensions of operator workload. Hum Factors 30: 95-110

Fukuba Y, Sato H, Sakiyama T, Yamaoka Endo M, Yamada M, Ueoka H, Miura A, Koga S (2009) Autonomic nervous activities assessed by heart rate variability in pre- and postadolescent Japanese. J Physiol Anthropol 28: 269-273 
Gould KS, Roed BK, Saus ER, Koefoed VF, Bridger RS, Moen BE (2009) Effects of navigation method on workload and performance in simulated high-speed ship navigation. Appl Ergon 40: 103-114

Hart SG, Stavel LE (1988) Development of NASA-TLX (Task Load Index): Results of empirical and theoretical research. In Hancock PA, Meshkati N eds. Human mental workload. North-Holland, Amsterdam, 139-183

ISO 10075-2 (1996) Ergonomic principles related to mental workload-Part 2: Design principles

ISO 10075-3 (2004) Ergonomic principles related to mental workload-Part 3: Principles and requirements concerning methods for measuring and assessing mental workload

Jacobs J, Embree P, Glei M, Christensen S, Sullivan P (2004) Characterization of a novel heart and respiratory rate sensor. Conf Proc IEEE Eng Med Biol Soc 3: 2223-2226

JIS C 6802 (2011) Safety of laser products

Johannsen G, Moray N, Pew R, Rasmussen J, Sanders A, Wickens CD (1979) Final report of experimental psychology group. In Moray $\mathrm{N}$ ed. Mental workload. Its theory and measurement. Plenum Press, New York, 101-114

Kobayashi H (2007) Inter- and intra-individual variations of heart rate variability in Japanese males. J Physiol Anthropol 26: $173-177$

Lohman B, Boric-Lubecke O, Lubecke VM, Ong PW, Sondhi MM (2001) A digital signal processor for Doppler radar sensing of vital signs. IEEE Eng Med Biol Mag 21: 161-164

Matsui T, Hagisawa K, Ishizuka T, Takase B, Ishihara M, Kikuchi M (2004) A novel method to prevent secondary exposure of medical and rescue personnel to toxic materials under biochemical hazard conditions using microwave radar and infrared thermography. IEEE Trans Biomed Eng 51: 2184-2188

Matsui T, Gotoh S, Arai I, Hattori H, Fujita M, Obara K, Masuoka K, Nakamura S, Takase B, Ishihara M, Kikuchi M (2006) Noncontact vital sign monitoring system for isolation unit (Casualty Care System). Mil Med 171: 639-643

Matsui T, Suzuki S, Ujikawa K, Usui T, Gotoh S, Sugamata M, Abe S (2009) The development of a non-contact screening system for rapid medical inspection at a quarantine depot using a laser Doppler blood-flow meter, microwave radar and infrared thermography. J Med Eng Technol 33: 481-487

Matsui T, Hakozaki Y, Suzuki S, Usui T, Kato T, Hasegawa K, Sugiyama Y, Sugamata M, Abe S (2010) A novel screening method for influenza patients using a newly developed noncontact screening system. J Infect 60: 271-277

Miyake S (2001) Multivariate workload evaluation combining physiological and subjective measures. Int J Psychophysiol 40: 233-238

O'Rourke MF, Kelly RP, Avolio AP (1992) The arterial pulse. Lea \& Febiger, Philadelphia
Princi T, Parco S, Accardo A, Radillo O, DeSeta F, Guaschino $S$ (2005) Parametric evaluation of heart rate variability during the menstrual cycle in young women. Biomed Sci Instrum 41: 340-345

Sakuragi S, Sugiyama Y (2009) Effects of reward and punishment on task performance, mood and autonomic nervous function, and the interaction with personality. $\mathrm{J}$ Physiol Anthropol 28: 181-190

Singh N, Mironov D, Armstrong PW, Ross AM, Langer A (1996) Heart rate variability assessment early after acute myocardial infarction. Pathophysiological and prognostic correlates. Circulation 93: 1388-1395

Sirevaag EJ, Kramer AF, Wickens CD, Reisweber M, Strayer DL, Grenell JF (1993) Assessment of pilot performance and mental workload in rotary wing aircraft. Ergonomics 36: 1121-1140

Suzuki S, Matsui T, Imuta H, Uenoyama M, Yura H, Ishihara M (2008a) A novel autonomic activation measurement method for stress monitoring: Non-contact measurement of heart rate variability using a compact microwave radar. Med Biol Eng Comput 46: 709-714

Suzuki S, Sumi K, Matsubara M (2008b) Cardiac autonomic control immediately after exercise in female distance runners. J Physiol Anthropol 27: 325-332

Suzuki S, Matsui T, Kawahara H, Ichiki H, Shimizu J, Kondo Y, Gotoh S, Yura H, Takase B, Ishihara M (2009) A noncontact vital sign monitoring system for ambulances using dual-frequency microwave radars. Med Biol Eng Comput 47: 101-105

Thijs JAJ, Muehlsteff J, Such O, Pinter R, Elfring R, Igney CH (2005) A Comparison of Continuous Wave Doppler Radar to Impedance Cardiography for Analysis of Mechanical Heart Activity. Conf Proc IEEE Eng Med Biol Soc: 3482-3485

Uenoyama M, Matsui T, Yamada K, Suzuki S, Takase B, Suzuki S, Ishihara M. (2006) Non-contact respiratory monitoring system using a ceiling-attached microwave antenna. Med Biol Eng Comput 44: 835-840

Vincent A, Craik FI, Furedy JJ (1996) Relations among memory performance, mental workload and cardiovascular responses. Int J Psychophysiol 23: 181-98

Wang F, Tanaka M, Chonan S (2006) Development of a wearable mental stress evaluation system using PVDF film sensor. J Adv Sci 18: 170-173

Received: May 17, 2011

Accepted: September 8, 2011

Correspondence to: Satoshi Suzuki, 3-3-35 Yamatecho, Suita, Osaka 564-8680, Japan

Phone: +81-6-6368-0915

Fax: +81-6-6388-8785

e-mail: ssuzuki@kansai-u.ac.jp 